thermal optima or increased heat tolerance over evolutionary time, and that is the pattern that is emerging.

Studies providing evidence for genetic change in response to recent, rapid climate change have come from research groups that have focused their efforts on one or a few species over several decades. These studies have involved retrospective comparisons in flies, mosquitoes, and blackcaps or have used pedigree analysis through multiple generations in squirrels and great tits. The time scales over which genetic changes are detectable cover a wide range. In mosquitoes, a clear change could be seen over 5 years (15). Moderate changes were detectable over 10 years in red squirrels (8). In great tits, even after 30 years, only the portion of the population that is most able to modify the timing of egg laying in response to earlier springs has changed genetically (11). Despite both phenotypic and genetic changes in the ability of great tits to track the seasonal availability of caterpillars, the average lifetime reproductive success of the population as a whole is declining. The population cannot keep pace with environmental change and may be vulnerable to extinction (11). Hence, the ability to evolve in response to recent climate warming does not, in itself, ensure that a population will survive $(2,11,21)$.

As these examples show, the effects of rapid climate warming have penetrated to the level of the gene in a diverse group of organisms. These genetic changes in populations affect the timing of major life history events: when to develop, when to reproduce, when to enter dormancy, and when to migrate. Small animals with short life cycles and large population sizes will probably adapt to longer growing seasons and be able to persist; however, populations of many large animals with longer life cycles and smaller population sizes will experience a decline in population size or be replaced by more southern species. Questions remain about the relative rates of environmental and evolutionary change $(5,11,21,22)$. But it is clear that unless the long-term magnitude of rapid climate change is widely acknowledged and effective steps are taken to mitigate its effects, natural communities with which we are familiar will cease to exist $(2,22-24)$.

\section{References and Notes}

1. C. Parmesan, G. Yohe, Nature 421, 37 (2003).

2. R. Warren, in Avoiding Dangerous Climate Change, H. J. Schellnhuber et al., Eds. (Cambridge Univ. Press, Cambridge, 2006), chap. 11.

3. D. Berteaux et al., Integr. Comp. Biol. 44, 140 (2004).

4. T. L. Root et al., Nature 421, 57 (2003).

5. L. Hughes, Trends Ecol. Evol. 15, 56 (2000).

6. G.-R. Walther et al., Nature 416, 389 (2002).

7. Evidence for genetically-based shifts in a population include differences between populations of animals reared under identical conditions after years or decades of selection (flies, mosquitoes); pedigree analysis, which establishes the genetic basis of phenotypic change based on resemblance between relatives in succeeding generations (squirrels, great tits); and differences between subpopulations in migratory patterns that persist in labreared offspring (blackcaps).

8. D. Réale et al., Evolution 57, 2416 (2003).

9. S. Bearhop et al., Science 310, 502 (2005).

10. P. Berthold et al., Nature 360, 668 (1992).

11. D. H. Nussey, E. Postma, P. Gienapp, M. E. Visser, Science 310, 304 (2005).

12. F. Rodríguez-Trelles, M. A. Rodríguez, Evol. Ecol. 12, 829 (1998).

13. M. Levitan, Evol. Ecol. Res. 5, 597 (2003)

14. P. A. Umina, A. R. Weeks, M. R. Kearney, S. W. McKechnie, A. A. Hoffmann, Science 308, 691 (2005).

15. W. E. Bradshaw, C. M. Holzapfel, Proc. Natl. Acad. Sci. U.S.A. 98, 14509 (2001).

16. W. E. Bradshaw, P. A. Zani, C. M. Holzapfel, Evolution 38, 1748 (2004).

17. Intergovernmental Panel on Climate Change, Climate Change 2001: The Scientific Basis, J. T. Houghton et al., Eds. (Cambridge Univ. Press, Cambridge, 2001).

18. T. R. Karl, K. E. Trenberth, Science 302, 1719 (2003).

19. A. S. Danilevskii, Photoperiodism and Seasonal Development of Insects (Oliver \& Boyd, Edinburgh, 1965).

20. A. Addo-Bediako et al., Proc. R. Soc. London Ser. B 267 739 (2000).

21. M. Lynch, in Conservation Genetics: Case Histories from Nature, ]. C. Avise, J. L. Hamrick, Eds. (Chapman \& Hall, New York, 1996), chap. 15

22. C. D. Thomas et al., Nature 427, 145 (2004).

23. A. van Vliet, R. Leemans, in Avoiding Dangerous Climate Change, H. J. Schellnhuber et al., Eds. (Cambridge Univ. Press, Cambridge, 2006), chap. 12.

24. E. Kolbert, Field Notes from a Catastrophe: Man, Nature, and Climate Change (Bloomsbury, New York, 2006).

10.1126/science. 1127000

\title{
Toward Robots That Can Sense Texture by Touch
}

A compact, high-resolution touch sensor has been developed from a thin film. Incorporation of this sensor into robotic hands may substantially improve their dexterity.

\section{Richard Crowder}

$\mathrm{T}$ oday's state-of-the-art dexterous robotic hands cannot achieve tasks that most 6 year-old children can do without thinking, such as tie a shoelace or build a house of cards. The improvement of the manipulative capabilities of robotic hands requires advances in a wide range of technologies, including mechanics, actuators, sensors, and artificial intelligence. Many robots - such as NASA's Robonaunt (1) - have the dexterity required to perform some of the tasks that we take for granted, but replication of the full manipulative capabilities of the human hand is still years away.

A key advance needed for these new robots is the development of a sensor or set of sensors that can replicate the human sense of touch. Most robotic systems incorporate binary touch

The author is in the School of Electronics and Computer Science, University of Southampton, Southampton S017 1B], UK. E-mail: rmc@ecs.soton.ac.uk sensors - that is, sensors that can distinguish between touch or no touch. Many more sophisticated sensors have been discussed in the literature, but their take-up by industry is hampered by manufacturing challenges, in particular, the assurance of protection against the wear and tear found in the real world. In contrast, vision sensors are almost commonplace in many robotic systems (2).

The development of tactile sensors is one of the most difficult aspects of robotics. (A tactile sensor measures force and spatial information, whereas touch is technically just the force at a single point.) Many technologies have been explored, including a carbon-loaded elastomer, piezoelectric materials, and micro-electromechanical systems (3). Many designs exist, but few have moved from the research laboratory to become a commercial success. Those that have tend to be robust and easy to construct, but provide poor spatial resolution.

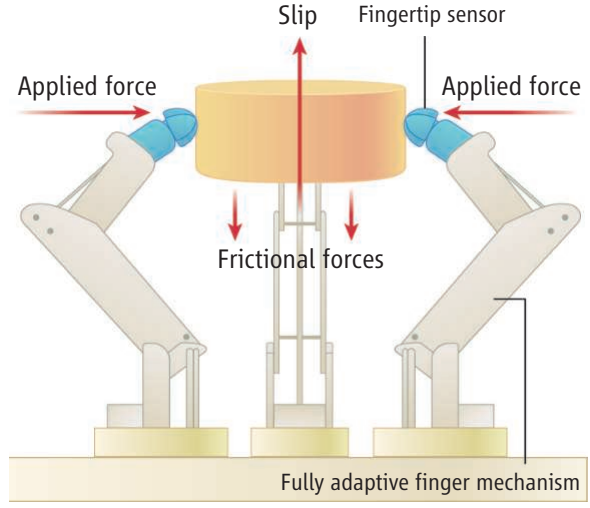

A three-fingered gripper. The fingertips are designed to allow a wide variety of objects to be grasped and manipulated. The addition of a fingertip sensor will not only allow the applied force to be controlled, but will also (with a suitable controller) minimize the object's slip in any direction. 
On page 1501 of this issue, Maheshwari and Saraf (4) report the development of a compact, high-resolution touch sensor. The thin-film design permits the authors to produce a single tactile sensor that is larger than a typical human fingertip, with similar resolution. However, as with most sensors of this resolution and size, the challenge is to extract the sensor's information efficiently - something that the human nervous system does with supreme efficiency.

The new sensor responds to an applied force either with electroluminescent emissions or with a change in current density. The electroluminescent response can be considered the high-resolution mode. The authors use a charge-coupled device (CCD) camera to capture the electroluminescent emissions from the sensor. This vision-based approach allows the high-resolution tactile image to be easily obtained and manipulated. The current density measurement, although of lower resolution, could also be used in a dexterous robot hand, either as a lower resolution sensor or for protection. Given that the sensing element is a film, it should be possible to use it to coat the fingertip or palm of a robot, although the CCD camera will need to be integrated with the sensing film.

The detection of texture at the resolution reported by Maheshwari and Saraf will allow not only object texture and local forces to be determined; slip can also easily be detected though examination of the tactile image over time. The early detection of slip is used in the control of many multifingered hands.

To appreciate the advantages of having a single sensor for both slip and texture, it is worth considering how it can be integrated into robotic hands that need to restrain and manipulate a wide range of objects under a wide range of conditions. To achieve a satisfactory grasp, optimal force control is required. Any movement of a robotic hand may result in the grasped object slipping and possibly being dropped; hence, the sensors on the hand have to register any slippage and adjust the applied forces to bring the object back to rest (see the figure).

The problem of defining the required grasp force is crucial and can be posed as an optimization problem (5). A range of techniques have been used to solve this problem. Some approaches are analytic solutions and cannot be easily implemented in real-time applications, particularly when dynamic adaptation to external disturbances is required. Also, analytic approaches cannot be used if variables such as object weight and hand acceleration are unknown. To overcome this situation, advanced control techniques have been developed, which use tactile sensors to measure the applied force to the object and its slip as required by the controller (6). Controllers that can optimize the applied force in response to slip have been successfully implemented $(7,8)$.

To achieve satisfactory control of a dexterous hand, a sensor requires a wide dynamic range, typically up to $1000 \mathrm{~Hz}$; the reported sensor is capable of achieving this. Dynamic application of the sensor is thus of considerable interest in the robotic community, and the high resolution of the tactile information will be of interest in medical robotics, particularly in minimal access (or keyhole) surgery.

The development of tactile sensors is one of the key technical challenges in advanced robotics and minimal-access surgery. The unique sensor developed by Maheshwari and Saraf could prove to be a key advance in technology, for reasons including relatively simple construction, apparent robustness, and high resolution. The next stage in the development of this sensor is to look at its robustness and performance characteristics over time. Once these issues are resolved, the sensor can be integrated into a dexterous hand, hopefully leading to an improvement in dexterity.

\section{References}

1. G. Huang, New Scientist 46 (4 February 2006).

2. M. Lee, Int. J. Robot. Res. 19, 636 (2000).

3. R. Crowder, in Handbook of Industrial Automation, R. Shell, E. Hall, Eds. (Dekker, New York, NY, 2000)، pp. 377-392.

4. V. Maheshwari, R. F. Saraf, Science 312, 1501 (2006).

5. A. Bicchi, V. Kumar, in Proceedings of IEEE International Conference on Robotics and Automation (IEEE, San Francisco, 2000), pp. 328-353.

6. M. Brown, C. Harris, Neurofuzzy Adaptive Modelling and Control (Prentice Hall International, Hemel Hempstead, UK, 1994).

7. J. Dominguez-Lopez, R. Crowder, R. Damper, C. Harris, in 2004 IEEE International Conference on Systems, Man and Cybernetics (IEEE, The Hague, Netherlands, 2004), vol. 4, pp. 3193-3198.

8. O. Fuentas, R. Nelson, in IEEE/SICE/RSJ International Conference on Multisensor Fusion and Integration for Intelligent Systems (IEEE, Washington, DC, 1996), pp. 342-348.

10.1126/science. 1129110

\section{ECOLOGY}

\section{Getting a Better Picture of the Ocean's Nitrogen Budget}

\section{Zbigniew S. Kolber}

The most common nitrogen-fixing cyanobacterium in the oceans is more abundant than previously estimated and can therefore account for at least a portion of the apparent oceanic nitrogen deficit.

$\mathrm{T}$ he deficit in the biologically available nitrogen supply required to support new production (photosynthetic activity that results in permanent carbon fixation) in tropical and subtropical oceans represents one of the most intriguing conundrums in oceanography. Upwelling of the deep, nutrient-rich waters through mechanisms of winter mixing due to storm activity, eddy pumping, and diffusion can supply only a fraction (40 to $60 \%$ ) of the necessary nitrate (1). The remainder must be provided by reduction of atmospheric dinitrogen $\left(\mathrm{N}_{2}\right)$, which requires breaking the ultrastable triple nitrogen-nitrogen bond. In the ocean, most of this process is performed by a nonheterocystous photosynthetic prokaryote, Trichodesmium sp. The estimates of nitrogen fixation by this diazotroph average $\sim 90 \mathrm{Tg} \mathrm{N}_{\text {year }}^{-1}$ (2), which represents less than $50 \%$ of the required new nitrogen $(3)\left(1 \mathrm{Tg}=10^{12} \mathrm{~g}\right)$. During the past 10 years, a substantial effort has been expended to account for the missing part in the ocean's nitrogen budget. Researchers have sought to reassess the diazotrophic activity of Trichodesmium or to identify other organisms that may fix the "miss-

The author is at the Monterey Bay Aquarium Research Institute, 7700 Sandholdt Road, Moss Landing, CA 95039, USA.E-mail: zkolber@mbari.org ing" nitrogen. Although new, single-cell diazotrophic bacteria have been identified (4), their potential contribution to nitrogen fixation has been difficult to quantify. On page 1517 of this issue, Davis and McGillicuddy (5) decided to have another "look" at this problem, literally. Using a towed undulating video camera, they captured images of Trichodesmium colonies (in puff and tuft morphologies; see the figure) over large areas of the Sargasso Sea. On the basis of the unexpectedly high abundance of these colonies, they postulate that the apparent nitrogen deficit in the world's oceans is simply due to an accounting error.

The video images of colonies paint a picture of Trichodesmium distribution in the water column very different from that described in the existing literature. Conventional net tows and bottle sample data indicate that Trichodesmium colonies generally congregate at the surface of the ocean, with their abundance sharply decreasing at depth. Such patterns of vertical distribution are consistent with (i) the prevalence of gas vacuoles within colonies of these cyanobacteria, which make them buoyant; (ii) the high energy requirements for bacterial nitrogenase to catalyze nitrogen fixation, which are met in well-lit surface waters; and (iii) independence from dissolved nitrate, which allows 